\author{
LA-UR- $-92-3587$ \\ $\therefore \quad$ DE93 003807
}

titLe POTENTIAL MCNP ENHANCEMENTS FOR NCT

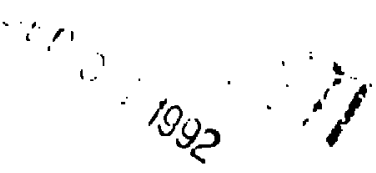

AUthoR(S) GuY P. Estes

William M. Taylor

SUBMitTED to Proceedings for Fifth International Symposium on Neutron

Capture Therapy for Cancer - Columbus, OH, September 13-17, 1992.

\title{
DISCLAIMER
}

This report wa prepared as an account of work spunsored by an agency of the IInited Siates Government. Neilher the United Situles Covernment nuf uny agency thereof, nur any of their employees, inakes uny warranty, express or implied, of ussumes uny legul liubility or responsibility for the uccuracy. completeness, or usefulness of uny information, apparatun, product, or process diaclosed, of reprenents that its uxe would not infringe privately owned pights. Reference herein (1) any specific commercial product, process, or service by trade name, trademart. manufucturef, or otherwice does not necessarily cunstitute or imply its endorsement, reomn. menduluon, of favoring by the Uniled Stalen (iovernment ir any agency thereaf. The viow end opinkins of authors expreanod herein do not necuasarily liate or reflect thoue of the Iniled Sitates (ioveramenl or any agency thereof.

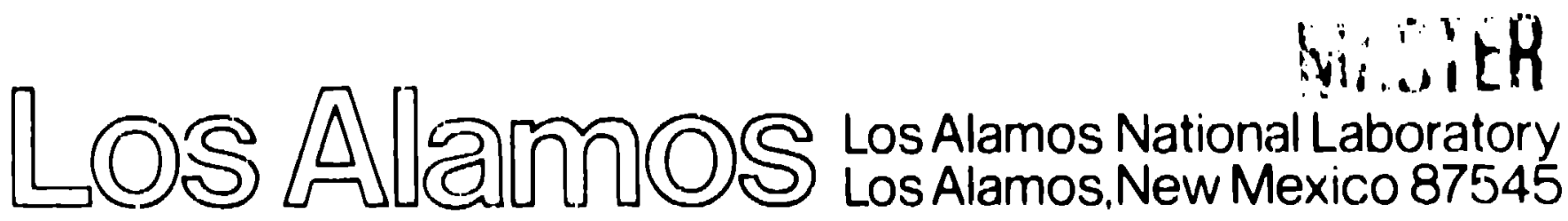




\section{POTENTIAL MCNP ENHANCEMENTS FOR NCT}

Guy P. Estes and William M. Taylor

Radiation Transport Group

Los Alamos National Laboratory

Los Alamos, NM 87545

\section{INTRODUCTION}

MCNP, a Los Alamo National Laboratory Monte Carlo radiation transport code, is currently widely used in the medical community for a variety of purposes including treatment planning, diagnostics, beam design, tolnographic studies, and radiation protection. This is particularly true in the Neutron Capture Therapy (NCT) community as evidenced by the numerous uses of MCNP in various publications. ${ }^{2}$ The current widespread medical use of MCNP after its general public distribution in about 1980 attests to the code's gener-' arsatility and usefulness, particularly since its development to date has not been influenced by medic . applications. This paper discusses enhancements to MCNP that could be implemented at Los Alas 10 for the benefit of the NCT community. These enhancements generally fall into two categories namely 1) thoee that have already been developed to some extent but are not yet publically available, and 2) those that seem both needed based on our current understanding of NCT goals, and achievable based on our working knowledge of the MCNP code.

MCNP is a general, coupled neutron/photon/electron Monte Carlo code developed and maintained by the Radiation Transport Group at Lo Alamos. It has been used extensively for radiation shielding studies, reactor anslysis, detector degign, physics experiment interpretation, oil and gas well logging, radiation protection studies, accelerator design, etc. over the years. It is estimated to have about 300 person-years of development effort to its credit, and traces it roots back to the World War II Manhattan Project and scientists such as Fermi, von Neumann, Ulam and others.

MCNP is a three-dimensional (3-D) geometry, continuous energy physics code capable of modeling complex geometries, specifying material regions s'lch as organs by the intersections of analytical surfaces. An example of the geometric compiexity posolible is shown in Fig. 1, where a partial MCNP model of the MIRD human phantom ${ }^{3}$ is presented. This figure is a SAERINA code $3-D$ picture of the MCNP model, where the flesh, ribs, and some organs have been omitted from the plot for clarity. This model will be discussed later in the context of potential medical imaging studies.

\section{NEAR-TERM ENHANCEMENTS}

There are a number of patches, techniques, etc. that have been dereloped in support of Lon Alamm programs or for one-of-a-kind studies that have never been incorporated into MCNP becallse of Incli vi prots:anmatic incentive. Most of this work esists in the form of patches which may or may not be consistent with the curtent version of MCNP, or as options in other codes. Fixamples of this category discussed belc'w are the pinhole "camera" imaging capability, the lattice geometry capability, and the MCNP/SABRINA particle track display capability. While some of these capabilities already 


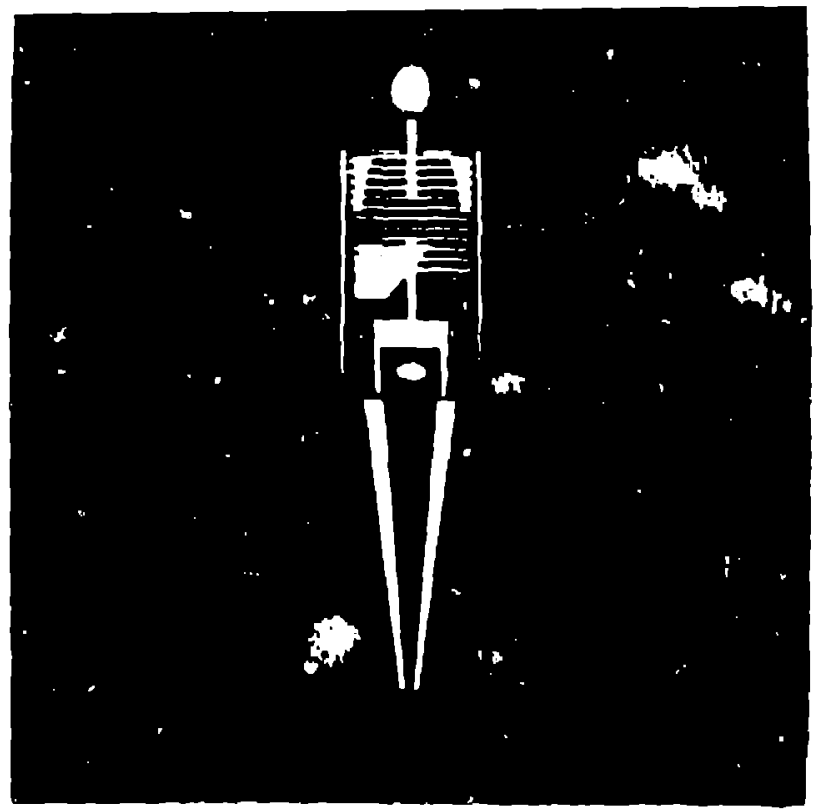

Figure 1. SABRINA-generated thre-dimenaiond plot of the MCNP geomotry model for the MIRD human phantom (some tronew and organe not shown).

exist informally, getting them into the public code would require a non-trivial amount of work because they would have to ve carefully integrated with all existing options in the code consiatent with our high quality assurance standards.

The first example given in this category is the pinhole "camera" imaging capability that could in principle be used to generate the equivalent of medical images, such as those produced by Anger cameras, uniformly redundant arrays, or coded apertures. Such images could be used for the purpose of helping interpret radioisotope diotributions and concentrations in regiona of the body from actual medical images. This capability is illustrated using the MIRD human phantom model of Fig. 1 Photon-enitling radioisotopes were asumed to be present in the major bones (skull, spine, arms, pelvis, and legs) of the model, and were imaged through and pinhole in an opaque medium as

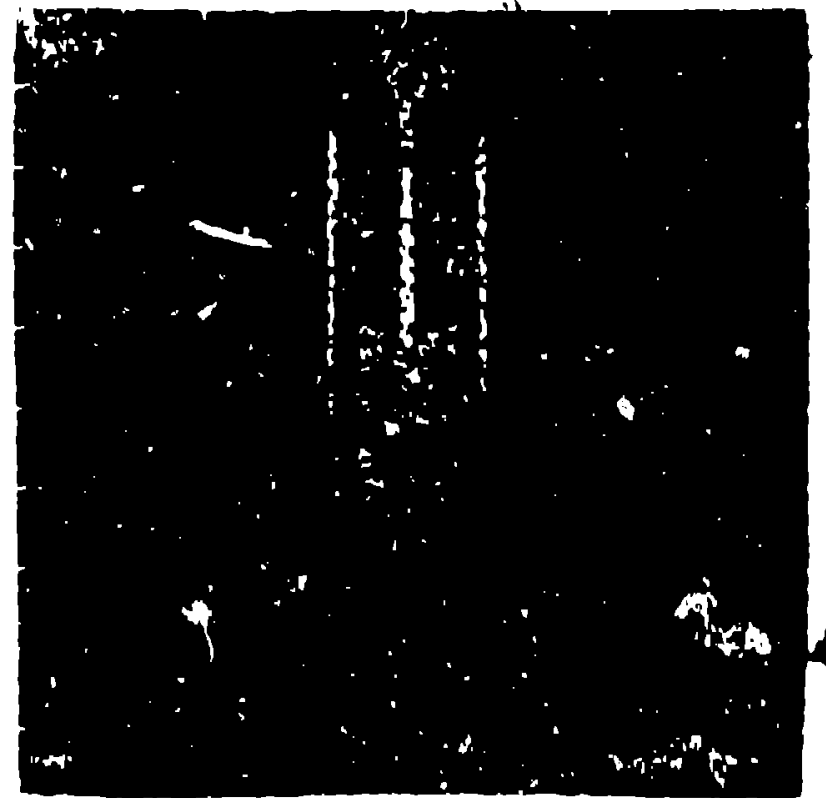

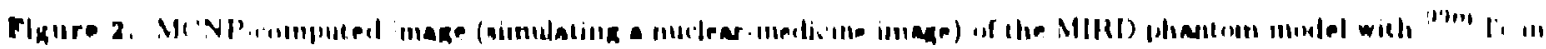

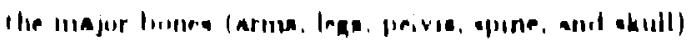


shown in Fig. 2. The bones with sources , tand out clearly in the figure, and the effect of scattered radiation in flesh and other bones can be seen in the interstitial regions. Since scattered and unscattered radiation $c$ an be segregated quite easily in MCNP, this technique could be used to computationally quantify scattered components in actual measured medical images, subtract them out, and thereby produce more unambiguous images for medical diagnoses. This will be discussed further in a later section in connection with creating patient-specific MCNP models from CT and MRI images.

The second potential near-term capability discussed is the use of the lattice capability, developed for reactor studies, to model the many cells needed for accurate dose calculations in the head (or body). It is maintained by s,ise radiation oncologists that more accuracy is needed in treatment planning to insure complete tumor kill, especially near material interfaces (e.g., brain/sinuses) where more approximate radiation transport calculations break down. This lattice geometry capahility has been tested for a brain geometry with several million cells, but work is needed to provide for automatically varying the material composition of each cell. An example of the geometry created is shown in Fig. 3 where the $0.15 \mathrm{~cm}$ mesh is typical of MRI scan resolutions.

The third neisr-term potential category is that of using the SABRINA particle track display car pability for qualitative scoping studies of neutron beam suitability for specific tumor treatment. The

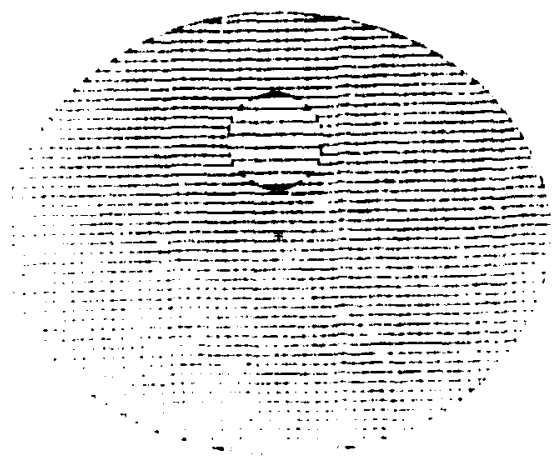

Figure 3. Plot of an MCNP gnometry with the ame voxel sise a e eypicel MRI ecan. The geom.try description required only 23 line of input wing the MCNP laftice option, including the simulated tumor.

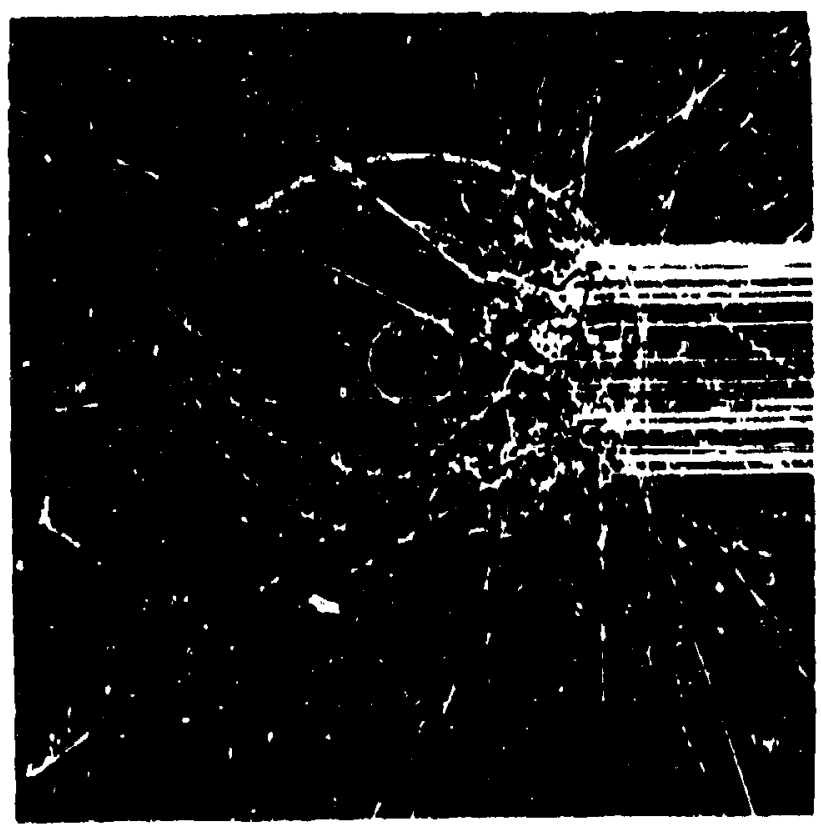

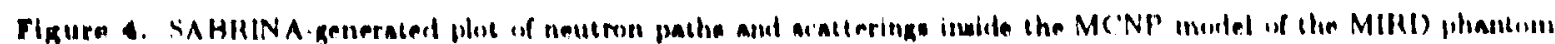
linend. 
MIRD phantom model head is shown in Fig. 4 with particle tracks shown. In this particular case, the energy of the incoming neutrons is too low, and most of them are thermalized before reaching the simulated tumor. Since this exampie was created with only 100 neutrons in the beam, such aualitative studies could be easily done in a quick scoping manner to determine the beam characteristics needed for a particular treatment scenario.

\section{POSSIBLE FUTURE ENHANCEMENTS}

With regard to longer range enhancements, it is felt that a number of new MCNP features could be developed that would be useful to the NCT community in the future. This aseartion is based on the fact that the rode was not developed with medical use in mind, and that improvements in efficiency, physics ancl ease of use for this purpose are reasonably attainable at Los Alamos based on our working knowledge of MCNY. The most altractive of these seems to be the potential for performing patient-specific MCNP ccmputations for diagnostic test interpretation and treatment scenarios, and for improving computational efficiency in order to perform near-real time treatment planning with these models.

By 1) generating patient-specific body geometry from CAT and/or MRJ scans and 2) simulating diagnostic images (e.g., CAT, Anger camera, SPECT) using these patient models, 3) high-accuracy dose distributions, radioisotope concentration assay, and scatter corrections would be possible for these patients. Such patient-specific calculations would undoubtedly improve the quality of care for patients, as well as lead to a more quantitative understanding of the effects of dose on tumors and healthy tissue function.

Ultimately, in order for MCNP to be used for routine clinical treatment planning, it will be necessary speed up the calculations by several orders of magnitude. 'This can be accomplished by several means. First, the regular rectangular mesh that results from creating patient-specific geometries from CT and MRI scans would be much simpler to calculate than the general geometry that is now built into MCNP. It has already been demonstrated at Los Alamos that providing special coding for regular mesh decreased computational time by a factor of 10 for similar problems. Secondly, computation time is becoming increasingly affordable, in particular with regard to desktop workstations. A Sun Sparc 2 now runs MCNP at about 1/4 the speed of one Cray YMP processor. The Sparc 10 that should be available in 1993 will be equivalent to about 4 Cray processors, a factor of 16 increase in computing power. Further advances are inevitable. Finally, Los Alamos and others are working on the "multi-tasking" of MCNP on workstations on a given network in order to fully utilize available C.PU's when the workstation owner is not doing so. At Los Alamos there is a cluster of 13 IBM RISC 6000's. whirh, when riulti-tasked with MCNP, will equal about 10 Cray processors.

\section{SUMMARY AND CONCLUSIONS}

In conciusion, it appears that patient-specific 3-D t.reatment planning and diagnosis is posaibie if a number of relatively straightforward improvements in MCNP can be implemented. Use of this inpability for near real-time therapy plauning should be practical in the near future when factoring in the likely advances in workstation computing power nud networking. With a courdinated efiort fro a the NC"T community, it should be possible to obtain support from DOE Defense Programs dunl.ulie? echnology teansfer initiatives for Los Alamos to provide the described MCNP enhancements described for the Ne"I" community.

\section{REFERENCES}

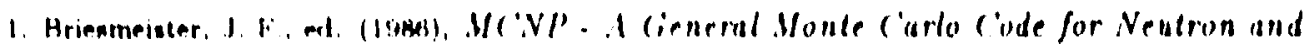

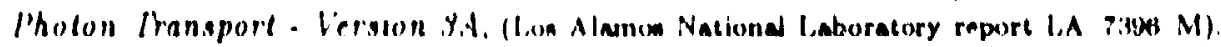

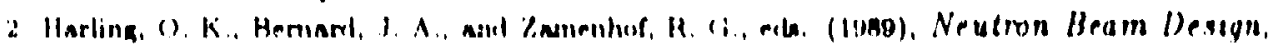
Cerelopment, and ferformance for. Veutron Ciplume l'herapy. (Plenum Prene. New Vurk)

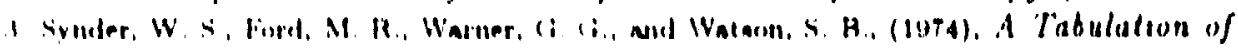

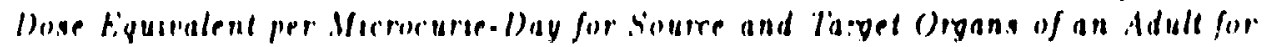

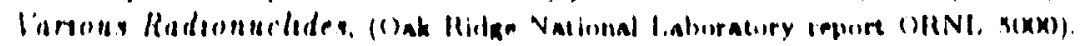

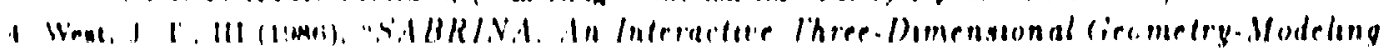

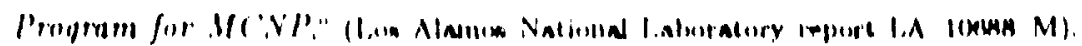


MIRD phantom model head is shown in Fig. 4 with particle tracks shown. In this particular case, the energy of the incoming neutrons is too low, and most of them are thermalized before reaching the simulated tumor. Since this example was created with only 100 neutrons in the beam, such qualitative studies could be easily done in a quick scoping manner to determine the beam characteristics needed for a particular treatment scenario.

\section{POSSIBLE FUTURE ENHANCEMENTS}

With regard to longer range enhancements, it is felt that a number of new MCNP features could be developed that would be useful to the NCT community in the future. This assertion is based nn the fact that the code was not developed with medical use in mind, and that improvernents in efficiency, physics and ease of use for this purpose are reasonably attainable at Los Alamos based on our working knowledge of MCNP. The most attractive of these seems to be the potential for performing patient-specific MCNP computations for diagnostic test interpretation and treatment scenarios, and for improving computational efficiency in order to perform near-real time treatment planning with these models.

By 1) generating patient-specific body geometry from CAT and/or MRI scans and 2) simulating diagnostic images (e.g., CAT, Anger camera, SPECr) using these patient models, 3) high-accuracy dose distributions radioisotope concentration assay, and scatter corrections would to possible for these patients. Such patient-specific calculations would undoubtedly improve the quality of care for patients, as well as lead to a more quantitative understanuing of the effects of dose on tumors and healthy tissue function.

Ultimately, in order for MCNP to be used for routine clinical treatment planning, it will be necessary speed up the calculations by several orders of magnitude. This can be accomplished by several means. First, the regular rectangular mesh that results from creating patient-specific geometries from CT and MRI scans would be much simpler to calculate than the general geometry that is now built into MCNP. It has already been demonstrated at Los Alamos that providing special coding for regular mesh decreased computational time by a factor of 10 for similar problems. Secondly, computation time is becoming increasingly affordable, in particular with regard to desktop workstations. A Sun Sparc 2 now runs MCNP at about 1/4 the speed of one Cray YMP processor. The Sparc 10 that should be available in 1993 will be equivalent to about 4 Cray processors, a factor of 16 increase in computing power. Further advances are inevitable. Finally, Los Alamos and others are working on the "multi-tasking" of MCNP on workstations on a given network in order to fully utilize available CPU's when the workstation owner is not doing so. At Lu Alamos there is a cluster of 16 IBM RISC 6000's, which, when multi-tasked with MCNP, wiil equal about 10 Cray processors.

\section{SUMMARY AND CONCLUSIONS}

in conclusion, it appears that patient-specific 3-D treatment planning and diagnosis is posyible if a number of relatively straightforward improvenents in MCNP can be implemented. Use of this capability for near real-time therapy planning should be practical in the near future when factoring in the likely advances in workstation computing power and netwoiking. With a coordinated effort from the NC.T community, it should he possible to ohtain support from DOE Defense Programs dual-use. tocnnology transfer initiatives for Los Alamos to provide the described MCSN enhancements described for the NC.T community

\section{REFERENCES}

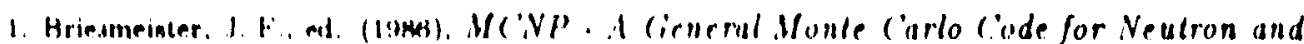

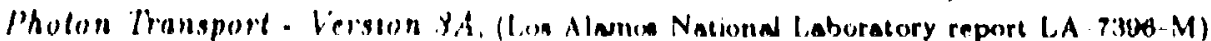

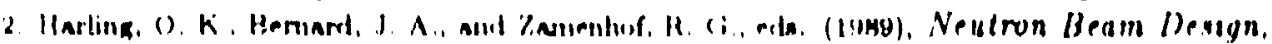

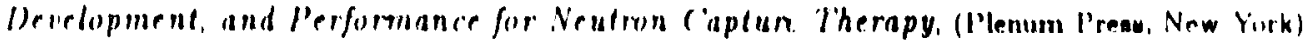

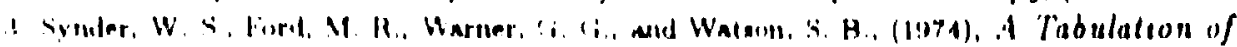

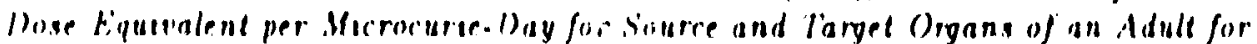

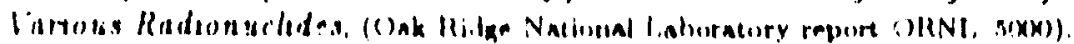

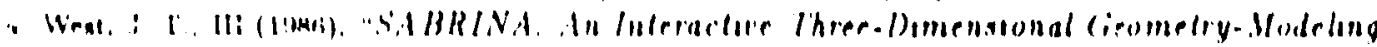

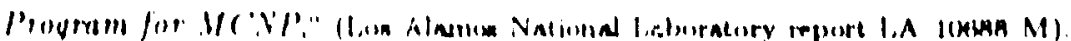

